\title{
Minimal Quantum Brownian Rectifiers ${ }^{\dagger}$
}

\author{
Igor Goychuk and Peter Hänggi* \\ Theoretische Physik I, Institut für Physik, Universität Augsburg, D - 86135 Augsburg, Germany \\ Received: January 11, 2001; In Final Form: March 16, 2001
}

\begin{abstract}
We consider directed quantum transport in one-dimensional, periodic tight-binding lattices that are driven by unbiased, but asymmetric driving forces which are composed of a fundamental and its second harmonic (harmonic mixing signal). The reduction to a single-band dynamics then yields a formally exactly integrable dynamics. An asymmetric drive generally breaks time-reversal symmetry without causing any dissipation. For an initially localized particle with zero momentum no finite coherent current emerges; in contrast, a directed current occurs, however, when prepared with an initial coherence. The presence of phase fluctuations yields a decaying current, thereby rendering the nondissipative currents a transient phenomenon. In contrast, the presence of quantum dissipation yields at all temperatures a finite asymptotic current independent of preparation.
\end{abstract}

\section{Introduction}

Statistical mechanics and quantum theory are two main pillars upon which most of the structure of theoretical physics is built. These two pillars considerably have influenced also the scientific "palmares" of Bruce Berne, who has advanced with many seminal contributions the fields of light scattering, transport theory in liquids, computer simulations of various molecular phenomena, and reaction rate theory. Quantum theory as well as statistical mechanics involve a time arrow as a primitive concept. In quantum mechanics one computes the probability amplitudes for possible measurable outcomes which follow specified initial preparations, i.e., not those which precede them. Likewise, in thermodynamics and statistical mechanics it is dogma that the total thermodynamic entropy of a closed system cannot decrease.

With this original work we elaborate on a problem that is rooted both in statistical mechanics and quantum theory. In particular, we focus on the question of obtaining in periodic structures a directed transport of matter, or, more generally, a transduction of information out of unbiased fluctuations. This topical concept is presently very much en vogue; it is known under labels such as Brownian rectifiers, ${ }^{1}$ Brownian motors, ${ }^{2-4}$ and born out from the challenge to obtain an understanding of intercellular transport along biological polymer filaments, also as molecular motors. ${ }^{4,5}$ Apart from its biological relevance this concept is enormously fruitful also for the construction of nanoscale and microscale devices, such as pumps, rectifiers, and particle separators. ${ }^{1,4}$ By use of a bottom-up approach, such motors have been synthesized recently even from single chiral molecules. ${ }^{6,7}$

For a Brownian rectifier to work the following two conditions seemingly must be obeyed: (i) the system must possess a source of asymmetry in the sense that it either exhibits an explicit asymmetric, periodic structure that breaks reflection symmetry, or it is driven by unbiased, but asymmetric forces. (ii) The system must operate far from equilibrium, because only then can it escape the limiting restriction imposed by the second law.

\footnotetext{
$\dagger$ Part of the special issue "Bruce Berne Festschrift". Dedicated to Bruce Berne on occasion of his 60th birthday.
}

If systems are reduced to the very conceptual backbone showing this two minimal requirements they present objects for studies from a fundamental point of view in their own right: clearly, this set up then mimics a Maxwell-Loschmidt demon ${ }^{8-10}$ which seemingly can be brought to life when operating in a stationary far from equilibrium situation. It is also generally believed that the occurrence of directed transport of information can only take place in the presence of irreversibility, or finite dissipation, respectively. Indeed, all known common schemes to model transport (Boltzmann equation, Fokker-Planck equations, Zubarev method, Kubo method, etc.) all assume the element of a time arrow. The use of dissipation is then a most natural way to break time-reversal invariance; such a violation presents a necessary (although not sufficient) element to induce directed current.

We emphasize, however, that the use of an asymmetric drive equally well does break time-reversal invariance. The dynamics is then governed by a pure Hamiltonian dynamics with a timeinhomogeneous Liouville-von Neumann evolution operator. This brings to us the area of Hamiltonian ratchets. ${ }^{11-13}$ Surprisingly to many of us, recent pioneering work by Sergej Flach and his collaborators, ${ }^{11}$ see also ref 12 , has shown that a nontrivial, directed current seemingly exists for Hamiltonian classical flows that exhibit both chaotic and regular solutions. The origin of such Hamiltonian directed classical currents has recently been traced back to the existence of a sum rule in phase space, ${ }^{13}$ namely, that the sum of corresponding invariant phase space volume times its average velocity for the union of invariant (ergodic) manifolds equals zero. In short, the overall directed current seemingly vanishes when averaged over all phase space. This still, however, allows for the possibility of a directed flow of information in a periodic system that starts out at some configurational position with initially vanishing velocity. It is this prerequisite which we shall assume throughout the rest of this work. To set the stage of our investigation, we note that even within this restriction of a zero initial velocity we should exclude some trivial situations that lead to a Hamiltonian directed current. Such a current occurs trivially if we take a bounded motion and transform it onto a moving frame (Galilei transformation with a nonzero, constant velocity). Likewise, a 
current occurs trivially with a biased initial momentum distributions. Yet another trivial situation occurs when the drive contains an initial bias in phase. For example, take the oscillatory driven dynamics

$$
\ddot{x}(t)=E \cos (\omega t+\psi)
$$

which after one integration yields with zero initial velocity, $\dot{x}(0)$ $=0$, the trivial solution for the velocity dynamics, i.e.,

$$
\dot{x}(t)=(E / \omega) \sin (\omega t+\psi)-(E / \omega) \sin (\psi)
$$

A time average then obviously yields a nonzero velocity whenever $\psi \neq 0$, $\bmod \pi$.

The occurrence of directed nondissipative current brings to mind a series of questions and problems. What is the role of quantum dynamics with its inherent smearing of phase space structures? What about a semiclassical treatment of the directed flow ${ }^{13}$ A driven Hamiltonian flow with a mixed phase space structure of dimension $d_{\text {phase-space }} \geq 5$ allows for Arnold diffusion among invariant manifolds. ${ }^{14}$ With the possibility that, generally, the mean time for mixing is not finite, ${ }^{15}$ the question then arises whether some seemingly stationary directed currents are in fact only extremely slow, power law like transients. In a first step, we shall next focus on the role of quantum fluctuations in a skeleton quantum system that possesses all the necessary minimal ingredients for directed quantum transport.

\section{Directed Quantum Current in Absence of Dissipation}

A. Deterministic Asymmetric Driving. To start, let us consider a charged particle such as an electron (with negative charge $e$ ) moving on a periodic lattice under the influence of a harmonic mixing electric field signal $E(t)$ of the form

$$
\mathrm{E}(t)=E_{1} \cos (\omega t+\psi(t))+E_{2} \cos (2 \omega t+2 \psi(t)+\phi)
$$

with the driving strengths $E_{1}, E_{2}$, frequency $\omega$, and the relative phase $\phi$. The quantity $\psi$ in eq 3 is the absolute phase of the two harmonics which generally can be a random function of time, describing the effect of dephasing. For the moment, however, we freeze this absolute phase to assume a fixed value. In this case, the presence of the nonzero absolute phase $\psi$ in eq 3 is equivalent to the time shift $t \rightarrow t+t_{0}$ with $t_{0}=\psi / \omega$. This fact makes obvious that the average over the randomly distributed phase $\psi$ is equivalent to an average over the initial time $t_{0}$ over a driving period $\mathcal{T}=2 \pi / \omega$.

The harmonic mixing signal in eq 3 constitutes the simplest kind of an asymmetric periodic field with a nonvanishing third moment $\left\langle\mathrm{E}^{3}(t)\right\rangle_{\omega}={ }^{3} /{ }_{4} E_{1}{ }^{2} E_{2} \cos \phi$. Here $\langle\ldots\rangle_{\omega}$ indicates the time average over the temporal period $\mathcal{T}$. Apart from the specific choice for the relative phase, i.e., $\phi=0, \bmod \pi$, the harmonic mixing signal violates time reversal symmetry, $t \rightarrow-t-2 t_{0}$, of the driven dynamics. Given such a violation of time-reversal symmetry one might expect, similar to the classical case, ${ }^{11,12}$ the emergence of a directed current even in the absence of dissipation.

In the absence of driving, the energy levels for the electron in the periodic potential possess a band structure. Let us simplify the situation further by restricting our analysis to the motion of the electron in the lowest energy band, neglecting thereby interband transitions. Then, in the representation of localized Wannier states, $|n\rangle$, the Hamiltonian of the driven quantum transport problem acquires the form ${ }^{17,18}$

$$
\begin{array}{r}
H_{\mathrm{TB}}(t)=-\frac{\hbar \Delta}{2} \sum_{n=-N}^{N}(|n\rangle\langle n+1|+| n+1\rangle\langle n|)- \\
e a \mathrm{E}(t) \sum_{n=-N}^{N} n|n\rangle\langle n|
\end{array}
$$

where $\hbar \Delta$ is the tunneling matrix element between neighboring states, $2 N+1$ denotes the number of sites, and $a$ is the lattice period. We shall describe the dynamics in terms of the density matrix $\rho_{n, m}$. By doing so, we go beyond the standard picture of pure Bloch states. The density matrix approach allows one to consider electrons prepared in mixed quantum-mechanical states as well. In the limit of an infinite $(N \rightarrow \infty)$ number of states this single-band tight-binding model is integrable and can be solved exactly for arbitrary external driving fields $\mathrm{E}(t) .{ }^{17,19,20}$ By the term "exactly" we mean that one can obtain an explicit analytical expression for the characteristic function, $F(k, t)=$ $\sum e^{i k n} \rho_{n, n}(t)$ (with $-\pi \leq k<\pi$ ), of the probability distribution $\rho_{n, n}(t)$ to find the electron on the site $n \cdot{ }^{20}$ Following the reasoning in ref 17, we find (in the limit $N \rightarrow \infty$ ) the result

$$
F(k, t)=\frac{1}{2 \pi} \sum_{n m} \rho_{n, n+m}(0) \int_{-\pi}^{\pi} d k^{\prime} e^{i\left\{\left[k^{\prime}-\eta(t, 0)\right] m+k n-\sum\left(k, k^{\prime}, t\right)\right\}}
$$

where

$$
\begin{aligned}
& \sum\left(k, k^{\prime}, t\right)=\Delta \int_{0}^{t}\left\{\cos \left[k^{\prime}-\eta(t, \tau)\right]-\right. \\
&\left.\cos \left[k^{\prime}-k-\eta(t, \tau)\right]\right\} \mathrm{d} \tau
\end{aligned}
$$

and

$$
\eta(t, \tau)=\frac{e a}{\hbar} \int_{\tau}^{t} \mathrm{E}\left(t^{\prime}\right) \mathrm{d} t^{\prime}
$$

With eq 5 at hand, any moment of the probability distribution $\rho_{n, n}(t)$ can be found from eqs $5-7$ by taking the corresponding number of derivatives. The first moment, $\langle x(t)\rangle=-i a F_{\mathrm{k}}^{\prime}(0, t)$, describes the mean position of the electron on the lattice, $\langle x(t)\rangle$ $=a \sum_{n} \rho_{n, n}(t)$. It reads ${ }^{20}$

$$
\langle x(t)\rangle=\langle x(0)\rangle+a|K| \Delta \int_{0}^{t} \mathrm{~d} \tau \sin [\eta(\tau, 0)+\varphi]
$$

with, $K=\sum_{n} \rho_{n, n-1}(0)$, being the coherence parameter and $\tan \varphi=\operatorname{Im} K / \operatorname{Re} K$. Next we introduce the quasi-momentum $p(t)$ obeying the so-called acceleration theorem, i.e., $\dot{p}(t)=e \mathrm{E}(t)$, with the pseudo-Hamiltonian given by ${ }^{18}$

$$
H(\langle x\rangle, p, t)=|K| \epsilon(p)-e\langle x\rangle \mathrm{E}(t)
$$

where $\epsilon(p)=-\hbar \Delta \cos (p a / \hbar)$ denotes the undriven energy spectrum. One can readily demonstrate that eq 8 provides the explicit solution of the driven nonlinear classical dynamics described with the pseudo-Hamiltonian $H(\langle x\rangle, p)$ for the initial quasi-momentum $p(0)=\hbar \varphi / a$ and the initial position $\langle x(0)\rangle$. These latter two quantities are defined by the initial density matrix $\rho_{n, m}(0)$. The relevant transport quantities of interest is the net quantum current

$$
j=e \lim _{t \rightarrow \infty} \frac{\langle x(t)\rangle}{t}
$$

being expressed here in a self-averaging manner. 
The result in eq 8 carries some truly remarkable consequences: for arbitrary external fields we find the prominent result that for a particle being initially prepared in a mixed state characterized by the diagonal density matrix $\rho_{n, m}(0)=$ $\rho_{n, n}(0) \delta_{n, m}$, implying a zero coherence parameter $K=0$; the mean particle position $\langle x(t)\rangle=\langle x(0)\rangle$ is not affected by the arbitrary driving fields. As a consequence we find that the current is identically zero ${ }^{20}$, i.e.,

$$
j=0
$$

This initial diagonal preparation mimics the classical situation of an initially localized particle. In clear contrast to the motion of a classical particle in a periodic potential ${ }^{11}$ exhibiting a mixed (chaotic/regular) phase space, the quantum mechanical motion of an initially localized particle-being restricted to the single band dynamics - does with $|K|=0$, i.e., an infinite effective mass - not exhibit an inertial dynamics, see eq 9. As a consequence, no net current is supported in this case.

Can a directed quantum current in absence of dissipation nevertheless be realized within a single band description? There is still given the possibility that with a finite coherence parameter $|K| \neq 0$, which induces a nonzero kinetic energy contribution, a finite current will emerge. To investigate this possibility we prepare the particle in a pure initial state give by the Bloch wave $|\Psi(0)\rangle=\sum_{n} c_{n}|n\rangle$, where $c_{n}=(1 / \sqrt{2 \mathrm{~N}+1}) e^{i p(0) n a / \hbar}$. For this case, $\rho_{n, m}(0)=c_{n} c_{m}^{*}$ and the coherence parameter is maximal, i.e., $|K|=1(N \rightarrow \infty)$. Note that, in the absence of the external driving, eq 8 indicates that any such Bloch state trivially carries a current, i.e., $j=e\langle\dot{x}(t)\rangle=e a \Delta \sin (p(0) a / \hbar)$. In view of our stated prerequisite in the Introduction, we consider here the case that the initial current is zero, yielding $p(0)=0$. Then, the tightbinding dynamics driven by a harmonic mixing signal with fixed relative phase, eq 3 , yields a finite limit in eq 10. The final main result for the time-averaged current in the absence of dissipation emerges as

$$
\begin{aligned}
\mathrm{j}=e \Delta a \sum_{k=-\infty}^{\infty} J_{2 k}\left(\xi_{1}\right) J_{k}\left(\xi_{2} / 2\right) \times & \\
& \sin \left[k \phi-\xi_{1} \sin (\psi)-\frac{1}{2} \xi_{2} \sin (2 \psi+\phi)\right]
\end{aligned}
$$

where $\xi_{1,2}=e a E_{1,2} /(\hbar \omega)$. This result holds for frozen fixed phase $\psi(t):=\psi$, cf. eq 3 .

In the lowest first order of the electric field amplitudes we find from eq 12

$$
j \approx-e \Delta a\left[\xi_{1} \sin \psi+\frac{1}{2} \xi_{2} \sin (2 \psi+\phi)\right]
$$

Alternatively, one can re-derive eq 13 by use of the effective mass approximation in the pseudo-Hamiltonian (eq 9): we replace the kinetic energy term with $p^{2} /\left(2 m^{*}\right)$, where $m^{*}=$ $\hbar /\left(|K| a^{2}\right)$ denotes the effective mass. The origin of current, $\langle\dot{x}\rangle$, in eq 13 is then obviously due to an initial phase effect as induced by the finite integration constant in $\langle\dot{x}\rangle$ in terms of nonzero values for $\psi$ and/or $\phi$ of the switched on driving field $\mathrm{E}(t)$. As such, it has nothing to do either with the nonlinear dynamics, nor with the violation of time-reversal symmetry for $\phi \neq 0$. This effect exists even in the case of a single harmonic driving, with either $\xi_{1}=0$, or $\xi_{2}=0$, see eq 2 . In absence of any phase fluctuations this (coherent) current carries no dissipation and it fails to decohere. Nevertheless, if one assumes that the initial field phase $\psi$ is randomly distributed in the interval $[0,2 \pi]$ with the probability density $P(\psi)=1 /(2 \pi)$ it becomes obvious from eq 13 that the averaging over $\psi$ yields zero current independently of the relative phase shift $\phi$. Likewise, one can show that the same result holds, namely a zero averaged current, after averaging over the initial phase $\psi$ (or, equivalently, over the initial time $t_{0}=\psi / \omega$ ) in eq 12. This result is exact, and does not depend on the amplitudes of the driving field, $E_{1,2}$. Moreover, we next demonstrate that dynamical phase fluctuations $\psi(t)$ cause a decaying current as well.

B. Role of Dephasing. Let us consider a simple model of dichotomous Markovian fluctuations of the absolute phase $\psi(t)$. This model allows for an exact analytic treatment, i.e., we set

$$
\psi(t)=\psi_{0} \alpha(t)
$$

where $\psi_{0}$ denotes the amplitude of phase fluctuations and $\alpha(t)$ $= \pm 1$ is the dichotomous Markov process (DMP) with the stationary autocorrelation function

$\left\langle\alpha(t) \alpha\left(t^{\prime}\right)\right\rangle=\exp \left(-v\left|t-t^{\prime}\right|\right)^{21}$. The parameter $v$ is the mean frequency of random phase jumps and defines the dephasing time $\tau_{0}=1 / \nu$. Using the relations, $\cos [\psi(t)]=\cos \left(\psi_{0}\right)$ and $\sin [\psi t)]=\sin \left(\psi_{0}\right) \alpha(t)$, the external driving can be recast into the form

$$
\mathrm{E}(t)=\tilde{\mathrm{E}^{2}}(t)-\frac{\hbar}{e a} g(t) \alpha(t)
$$

where $\tilde{\mathrm{E}^{2}}(t)$ is given by eq 3 for $\psi(t)=0$, but with the renormalized amplitudes $E_{1} \rightarrow \tilde{E}_{1}=E_{1} \cos \left(\psi_{0}\right)$ and $E_{2} \rightarrow \tilde{E}_{2}$ $=E_{2} \cos \left(2 \psi_{0}\right)$. Moreover, the function $g(t)$ in eq 15 reads

$$
g(t)=g_{1} \sin (\omega t)+g_{2} \sin (2 \omega t+\phi)
$$

where the amplitudes $g_{1}=e a E_{1} \sin \left(\psi_{0}\right) / \hbar$ and $g_{2}=e a E_{2}$ $\sin \left(2 \psi_{0}\right) / \hbar$ have the dimension of a frequency.

In the presence of random phase fluctuations, the stochastically averaged time-dependent current is given by

$$
j(t)=e \frac{\mathrm{d}\langle\langle x(t)\rangle\rangle_{\psi}}{\mathrm{d} t}
$$

where the outer average $\langle\ldots\rangle_{\psi}$ denotes the average over the phase fluctuations. The stationary current then is given by $j=\lim _{t \rightarrow \infty}$ $j(t)$. For the most interesting case with a coherence parameter of $|K|=1$, it follows from eq 8 that

$$
j(t)=e a \Delta \operatorname{Im}\left\{\exp (i[\tilde{\eta}(t, 0)+p(0) a / \hbar])\langle U(t)\rangle_{\psi}\right\}
$$

where $\tilde{\eta}(t, 0)$ is defined in eq 7 , but with $\mathrm{E}^{\sim}(t)$ taken from eq 15 . The function $\left\langle U(t)_{\psi}\right\rangle=\left\langle\exp \left[-i \int_{0}^{t} \alpha(\tau) g(\tau) \mathrm{d} \tau\right]\right\rangle_{\psi}$ in eq 18 is the averaged solution of the auxiliary stochastic differential equation

$$
\dot{U}(t)=-i g(t) \alpha(t) U(t)
$$

which describes a generalized Kubo oscillator ${ }^{21}$ with the stochastic frequency $\Omega(t)=g(t) \alpha(t)$. The averaged solution of eq 19 can be written by virtue of the Floquet theorem in the form

$$
\langle U(t)\rangle_{\psi}=e^{-\Gamma_{1} t} u_{1}(t)+e^{-\Gamma_{2} t} u_{2}(t)
$$

with positive valued Floquet values $\Gamma_{1,2}>0$, and time-periodic Floquet modes $u_{1,2}(t+2 \pi / \omega)=u_{1,2}(t)$. It then follows from eq 18 that the smallest of the two Floquet exponents, $\Gamma=$ $\min \left\{\Gamma_{1}, \Gamma_{2}\right\}$, in eq 20 characterizes the time scale on which some (transient) current can exist. Even if the current exists in the absence of phase fluctuations $\psi(t)$, it will relax in real life 
situations for times $t \gg \Gamma^{-1}$. This result that in the presence of a random driving a stationary $(t \rightarrow \infty)$ current cannot be realized in absence of dissipation has been shown by us previously in ref 20. Next, we shall evaluate this time scale explicitly.

The averaged solution of eq 19 with $g(t)=$ const has been given by Kubo. ${ }^{22}$ It can also be looked up in the book by Van Kampen. ${ }^{21}$ In our case, the problem is more intricate. To solve the task we apply the formalism of the so-termed "formulas of differentiation" 23 to yield the coupled set of differential equations

$$
\begin{gathered}
\frac{\mathrm{d}}{\mathrm{d} t}\langle U(t)\rangle_{\psi}=-i g(t)\langle\alpha(t) U(t)\rangle_{\psi} \\
\frac{\mathrm{d}}{\mathrm{d} t}\langle\alpha(t) U(t)\rangle_{\psi}=-v\langle\alpha(t) U(t)\rangle_{\psi}-i g(t)\langle U(t)\rangle_{\psi}
\end{gathered}
$$

for the average $\langle U(t)\rangle_{\psi}$ and the correlation $\langle\alpha(t) U(t)\rangle_{\psi}$. The initial conditions in eq 21 follow as $\langle U(0)\rangle_{\psi}=1$ and $\langle\alpha(0) U(0)\rangle_{\psi}=$ 0 . From here on, sailing becomes smooth by observing that the set of eqs 21 is indeed equivalent to a Hill equation for $\langle U(t)\rangle_{\psi}$. To solve eq 21, we use the transformation $\langle U(t)\rangle_{\psi}=r(t)$ $\cos [\Phi(t) / 2],\left\langle\alpha(t) U(t)_{\psi}=\operatorname{ir}(t) \sin [\Phi(t) / 2]\right.$, and end up with

$$
\begin{gathered}
\dot{r}(t)=-v \sin ^{2}(\Phi(t) / 2) r(t) \\
\dot{\Phi}(t)=-v \sin [\Phi(t)]-g(t)
\end{gathered}
$$

for the new variables $r(t)$ and $\Phi(t)$. The initial conditions transform into $r(0)=1$, and $\Phi(0)=0$. In terms of the unknown solution $\Phi(t)$, eq 22 yields for the averaged solution of the stochastic differential eq 19 the result

$$
\langle U(t)\rangle_{\psi}=\exp \left\{-v \int_{0}^{t} \sin ^{2}[\Phi(\tau) / 2] d \tau\right\} \cos [\Phi(t) / 2]
$$

This formal expression holds for an arbitrary function $g(t)$ and is not restricted to the class of periodic functions only. Because the second equation in eqs 22 cannot be integrated in closed form for $g(t)$ given in eq 16, the solution $\langle U(t)\rangle_{\psi}$ remains implicit. However, one can deduce the corresponding decay rate $\Gamma$ in an analytical form for relevant limiting cases. The most interesting situation is the case of a highly coherent field with small field strengths such that $v \ll \omega$ and $g_{1,2} \ll \omega$. In this case, the amplitude of oscillations of $\Phi(t)$ is small, $\Phi(t) \ll 1$, and one can expand $\sin [\Phi(t)] \approx \Phi(t)$ in eqs 22 . After some straightforward calculations, we obtain in the lowest order in the driving field strengths for the rate

$$
\Gamma=\frac{1}{8} v\left\{\sin ^{2}\left(\psi_{0}\right)\left(\frac{e a E_{1}}{\hbar \omega}\right)^{2}+\sin ^{2}\left(2 \psi_{0}\right)\left(\frac{e a E_{2}}{2 \hbar \omega}\right)^{2}\right\}
$$

implying for the transient current (for $t \gg v^{-1}$ ) the central result

$$
j(t)=j \exp (-\Gamma t)
$$

with $j$ is given in eq 13 with $\psi=0$.

This result inherits the following consequences. (i) A nonzero stationary current does not exist. (ii) The decay rate $\Gamma$ for the transient current is determined by the mean rate of phase fluctuations $v$, the amplitude of the phase fluctuations $\psi_{0}$, and also by the intensities of the field components $e a E_{1,2} / \hbar \omega$. (iii) With the increasing field strength, not only does the fieldinduced current $j$ increase, cf. eq 13 but also at the same time the current decay rate is also enhanced, cf. eq 24. Moreover, even if an arbitrary initial current was present it will decay with the rate of eq 24, as it follows from eq 18.

To decide whether the coherent field induced current is asymptotically finite or only transient, one can put forward the following criterion: (1) introduce small phase fluctuations $\psi(t)$ in the otherwise strictly periodic field $\mathrm{E}(t)$, cf. eq 3 , and (2) evaluate the limits in the sequence

$$
j=e \lim _{v \text { or } \psi_{0} \rightarrow 0} \lim _{t \rightarrow \infty} \frac{\mathrm{d}\langle\langle x(t)\rangle\rangle_{\psi}}{\mathrm{d} t}
$$

The order of limits in eq 26 is very important and cannot be interchanged. The application of this criterion to the quantum current in the absence of dissipation yields within a single-band description $j=0$, meaning that no finite stationary contribution survives. Whether this conclusion survives in the full potential picture, i.e., beyond the single-band description, still remains an open question. The authors presume, however, that indeed this is the case.

\section{Stationary Quantum Current in the Presence of Dissipation}

In the previous section we demonstrated that the field-induced current is at most a transient phenomenon under realistic conditions. The role of finite dissipation is thus crucial to produce possibly nonvanishing, stationary directed currents. Directed net currents in simple tight-binding models that are generated by the combined action of dissipation and external driving has been addressed in refs 16,20 , and 24 by use of different approaches. It is interesting to note that the coupling to a thermal bath in the deep cold, i.e., at the absolute zero temperature $T=0$ is already sufficient to produce a nonvanishing current. This shows that a directed current can emerge due to a cooperative rectification of zero-point quantum fluctuations and external unbiased driving. Let us elucidate this interesting point in further detail. We adopt here the conventional model of quantum dissipation, i.e., we couple the quantum particle bilinearly to a thermal bath of harmonic oscillators ${ }^{3,25,26}$

$$
H_{\mathrm{B}}=\frac{1}{2} \sum_{i}\left[\frac{\hat{p}_{i}^{2}}{m_{i}}+m_{\mathrm{i}} \omega_{i}^{2}\left(\hat{x}_{i}-\frac{c_{i}}{m_{\mathrm{i}} \omega_{i}^{2}} \hat{x}\right)^{2}\right]
$$

where $\hat{x}=a \sum_{n} \mathrm{n}|n\rangle\langle n|$ is the position operator of quantum particle. The bath influence on the quantum particle is fully captured by the Gaussian thermal force operator $\hat{F}(t)=$ $\sum_{i} c_{i} \hat{x}_{i}(t)$, possessing the complex-valued autocorrelation function

$$
\langle\hat{F}(t) \hat{F}(0)\rangle_{\text {eq }}=\frac{\hbar}{\pi} \int_{0}^{\infty} J(\omega) \frac{\cosh \left(\hbar \omega / 2 k_{\mathrm{B}} T-i \omega t\right)}{\sinh \left(\hbar \omega / 2 k_{\mathrm{B}} T\right)} \mathrm{d} \omega
$$

Here, $J(\omega)=\pi / 2 \sum_{i} c_{i}^{2} / m_{i} w_{i} \delta\left(\omega-\omega_{\mathrm{i}}\right)$ denotes the bath spectral density which will be taken in the Ohmic form $J(\omega)=$ $\eta \omega e^{-\omega / \omega_{c}}$, with cutoff frequency $\omega_{\mathrm{c}} \gg \Delta$ and friction coefficient $\eta$. Then, the autocorrelation function $\left\langle\hat{F}(t) \hat{F}(0)_{e q}\right.$ can be evaluated exactly. ${ }^{25,26}$. In the limit $k_{\mathrm{B}} T \ll \hbar \omega_{c}$ it can be approximated by

$$
\langle\hat{F}(t) \hat{F}(0)\rangle_{\mathrm{eq}} \approx \frac{1 \quad \hbar \eta \omega_{\mathrm{c}}^{2}}{\pi_{\left(1+i \omega_{\mathrm{c}} t\right)^{2}}}+2 k_{\mathrm{B}} T \eta f(t)
$$

where

$$
f(t)=\frac{1}{2 \tau_{T}}\left[\frac{\tau_{T}^{2}}{t^{2}}-\frac{1}{\sinh ^{2}\left(t / \tau_{T}\right)}\right]
$$

is a function that assumes a Dirac $\delta$-function in the limit $\tau_{T}=$ $\hbar /\left(\pi k_{\mathrm{B}} T\right) \rightarrow 0$. In eq 29 , the first (complex-valued) term 
corresponds to the zero-temperature contribution (due to the zero-point fluctuations) and the second contribution corresponds to the thermal fluctuations. The former one constitutes a genuine quantum-mechanical feature which is absent in the classical theory. The latter one corresponds to a classical Gaussian, nonMarkovian colored noise contribution with a thermal noise intensity $D=2 \eta k_{\mathrm{B}} T$ and an autocorrelation time scale $\tau_{T}$ which inherits quantum-mechanical features.

It is convenient to introduce the dimensionless friction coefficient $\alpha=\eta a^{2} /(2 \pi \hbar)$ (Kondo parameter). In ref 16, we have shown that in the incoherent transport regime, where the transport is dominated by incoherent quantum transitions among neighboring lattice sites, the quantum current is given by a Golden Rule type expression

$$
j=e a \Delta^{2} \int_{0}^{\infty} \mathrm{d} \tau \exp \left[-Q^{\prime}(\tau)\right] \sin \left[Q^{\prime \prime}(\tau)\right] \operatorname{Im}[\Phi(\tau)]
$$

where

$$
Q^{\prime}(t)+i Q^{\prime \prime}(t)=\frac{a^{2}}{\hbar^{2}} \int_{0}^{t} \mathrm{~d} t_{1} \int_{0}^{t_{1}}\left\langle\hat{F}\left(t_{2}\right) \hat{F}(0)\right\rangle_{\text {eq }} \mathrm{d} t_{2}+i \lambda t
$$

and

$$
\begin{aligned}
\Phi(\tau)= & \left\langle\left.\exp \left(i \frac{e a}{\hbar} \int_{t}^{t+\tau} \mathrm{E}\left(t^{\prime}\right) \mathrm{d} t^{\prime}\right)\right|_{\omega}=\right. \\
& \sum_{k=-\infty}^{\infty} J_{2 k}\left(2 \xi_{1} \sin (\omega \tau / 2)\right) J_{\mathrm{k}}\left(\xi_{2} \sin (\omega \tau)\right) \mathrm{e}^{-i k(\varphi+\pi / 2)}
\end{aligned}
$$

In eq $32, \hbar \lambda=a^{2} \int_{0}^{\infty} \mathrm{d} \omega J(\omega) / \pi \omega$ denotes the bath reorganization energy. ${ }^{27}$ The incoherent tunneling regime always occurs for $\alpha \geq 1$. For $\alpha<1$, the validity region of eq 31 is restricted (for small strengths and frequencies of the driving field) to sufficiently high temperatures. Note, however, that for a strong, high-frequency driving $\mathrm{E}(t)$ the accuracy of perturbation theory in the tunneling matrix element $\Delta$, which leads to the result in eq 31 , becomes drastically improved, cf. ref 28 . Thus, our result in eq 31 presents indeed a very good approximation even for $\alpha$ $<1$, except for the case of very small $\alpha$ and temperature $T$, when coherent tunneling begins to dominate.

One can show that the application of the criterion in eq 26 does not change the result in eq 31 . The current is stationary, i.e., it does not decay in time. As follows from eqs 31 and 33, the current vanishes identically, i.e., $j=0$, for $\phi=$ $\{\pi / 2,3 \pi / 2\}$, implying that all odd moments of the unbiased driving field, $\left\langle E^{2 n+1}(t)_{\omega}\right.$ are zero. This result is not perturbative in the driving field strength. Thus, the directed current appears in form of a nonlinear response to the external driving field if any odd moment of order $n \geq 3$ of the unbiased driving field, is different from zero. Indeed, for $T \neq 0$ the integrand in eq 31 decays exponentially in time. This circumstance makes the current an analytical function of the driving strengths, $E_{1}$ and $E_{2}$, respectively. As a consequence, in the lowest third order of the harmonic mixing driving strengths one finds that the dissipative result $j$ is proportional to

$$
j \propto\left\langle\mathrm{E}^{3}(t)\right\rangle_{\omega} \propto E_{1}^{2} E_{2} \cos (\phi)
$$

The nontrivial quantum prefactor involves the dissipation strength and the temperature, see in refs 16 and 24 .

The case of zero temperature is peculiar. In this case the current ceases to be an analytical function of the external field, thus an expansion to the lowest order in the field strengths fails. Nevertheless, a finite rectification current persists at $T=0$.
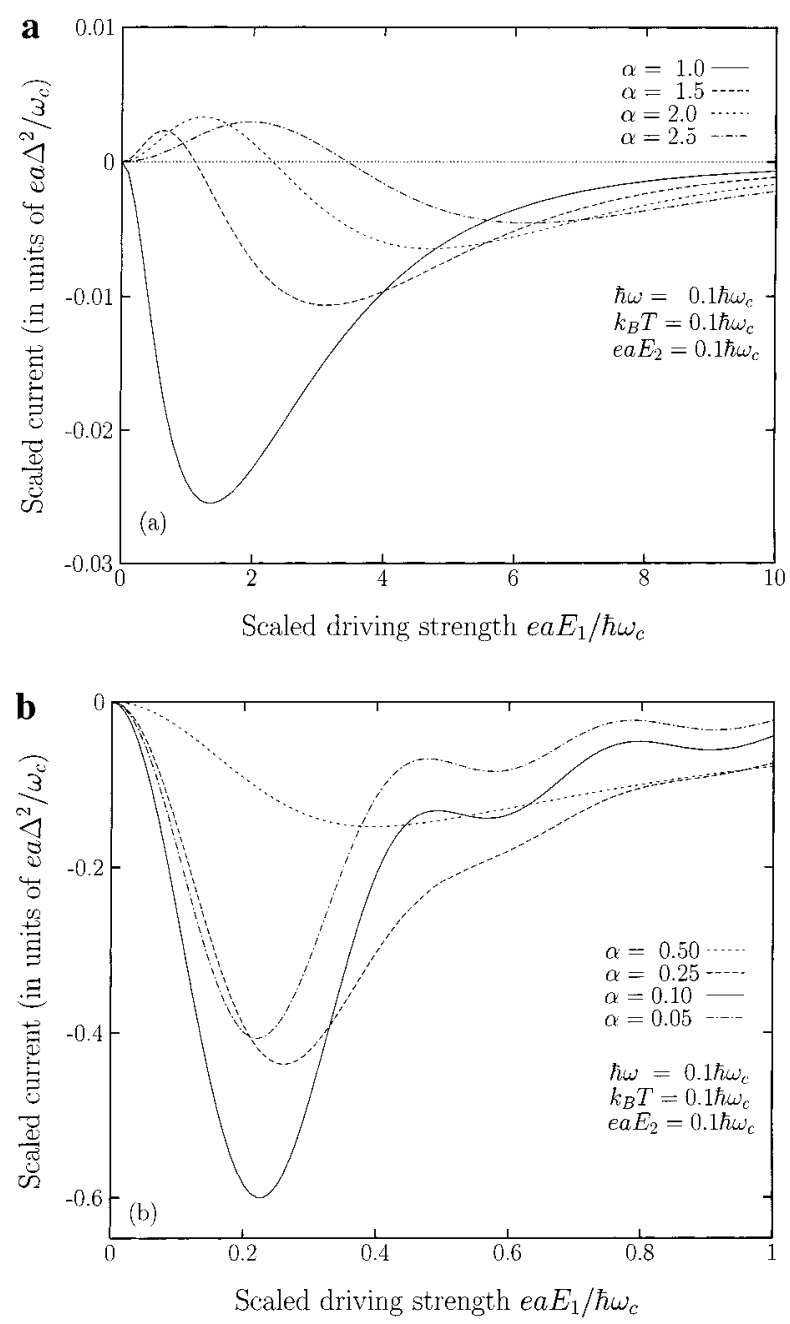

Figure 1. Directed current vs the strength of the fundamental harmonic in the harmonic mixing signal. The parameters used in calculations are given.

Unfortunately, the corresponding integral in eq 31 is too complicated and cannot be given in closed form. Some numerical calculations are depicted in Figure 1 for the case of adiabatic driving, $\omega \ll \omega_{\mathrm{c}}$, where the results become practically insensitive to the variations of the driving frequency $\omega$. Besides, we assumed that one of the driving harmonics is weak, $e a E_{2} \ll$ $\hbar \omega_{\mathrm{c}}$, and the temperature is small, $k_{\mathrm{B}} T \ll \hbar \omega_{\mathrm{c}}$. For the case in Figure 1, it was sufficient to take into account only the terms with $|k| \leq 1$ in the series 33 . As it can be deduced from Figure 1 the current becomes smaller with increasing friction strength $\alpha$. On the other hand, in accordance with the previous analysis and eq 31 , it also diminishes in the limit of small $\alpha \rightarrow 0$, cf. Figure $1 b$.

This implies that there exists an optimal value of the friction coefficient $\alpha$ which maximizes the current strength. This nontrivial effect is in the spirit of the so-called stochastic resonance (SR), ${ }^{29}$ where the addition of noise $\hat{F}(t)$ to the input driving signal $E(t)$ can optimize the current response. The considered system displays for $\alpha>1$ also a genuine SR behavior, when the friction strength $\eta$ is fixed and the thermal noise intensity $D=2 \eta k_{\mathrm{B}} T$ is varied. The increase of $D$ can also optimize the current value. ${ }^{16}$ Moreover, for $\alpha>1$ the current flows for small field amplitudes in the intuitively expected direction which is pointed out by the sign of $\left\langle E^{3}(t)\right\rangle_{\omega}$. With increasing driving strengths $E_{1}$, however, the current undergoes a reversal, i.e., it flows into the opposite, counter- 
intuitive direction. For $\alpha \leq 1$ this counterintuitive behavior dominates, cf. Figure 1b. Finally we note that for $\alpha \sim 0.1$ the current is by 1 order of magnitude larger than for $\alpha \sim 1$. Thus, the case of intermediate $\alpha \sim 0.1$ is preferable to observe the rectification current in practice. We also remark that the strength of the external driving should be optimized; notably the current diminishes for increasingly strong driving, cf. Figure 1.

\section{Conclusions}

In this work we have focused on the possibility to generate noise-induced directed quantum currents in periodic structures of minimal complexity; namely, the rectification of quantum noise in periodic tight binding lattices. The simplest way to obtain a finite directed current is by the combined use of both dissipation and nonequilibrium, asymmetric driving forces.

While dissipation breaks time-reversal symmetry in an obvious way, thereby breaking also detailed balance symmetry, it is not sufficient to yield finite transport. The key additional element is a source of dynamical asymmetry. This so generated directed current can also exhibit individual (possibly multiple) reversals as a function of varying system parameters. Such current reversals are of prime technological use: it opens the possibility for shuttling around into different directions species of quantum particles. At a point of net zero current the particles may become selected according to some internal parameter such as their initial energy, etc.; in this sense the device then operates as a quantum Maxwell demon away from thermal equilibrium. We have addressed with this work only the regime of incoherent quantum tunneling. A study of the difficult crossover behavior which involves on an equal footing coherent and incoherent driven tunneling events toward the case with no dissipation has yet to be undertaken.

Indeed, a most intriguing situation occurs when dissipation is abandoned altogether. As shown in section II, a finite, timeaveraged quantum current can occur in certain circumstances; but it will decay under realistic driving conditions when a sort of quantum nondemolition perturbations are acting. This has been exemplified in section II.B with a nondissipative dephasing mechanism. This topic of quantum Hamiltonian rectifiers then opens a Pandora's box for all kind of further complications. For example, the interplay of chaos and regular (classical) motion together with quantum ergodicity is well-known to be of relevance also for chemical reaction schemes, as pioneered with the isomerization reaction scenario by Berne and collaborators in the early eighties. ${ }^{30} \mathrm{~A}$ particular interesting situations arises when two, ${ }^{31}$ or a whole series of stadium billiards are coupled to form a one-dimensional array. The problem of energy transfer as well as the problem of rectification in terms of asymmetric temporal perturbations are expected to present interesting challenges, both in the classical regime and in the quantum regime, respectively. For example, the crossover from the full quantum toward the full classical behavior and the role of chaos as a substitute of dissipation influences are only beginning to be investigated in detail. The authors thus look forward to to seeing these concepts promoted further on the level of fundamental studies and also for practical applications in chemical and physical systems.

Acknowledgment. One of the authors (P.H.) herewith dedicates this work to Bruce Berne. Moreover, he thanks him for all those insightful and most fruitful discussions over the years (he learned a lot from him!). This work has been supported by Sonderforschungsbereich 486, Project A10.

\section{References and Notes}

(1) Hänggi, P.; Bartussek, R. In Nonlinear Physics of Complex Systems-Current Status and Future Trends; Parisi, J., Müller, S. C., Zimmermann, W., Eds.; Lecture Notes in Physics 476; Springer: Berlin, 1997; pp 294-308.

(2) Astumian, R. D. Science 1997, 276, 917.

(3) Reimann, P.; Hänggi, P. Chaos 1998, 8, 629.

(4) Reimann, P. Phys. Rep. In press; cond-mat/0010237.

(5) Jülicher, F.; Ajdari, A.; Prost, J. Rev. Mod. Phys. 1997, 69, 1269.

(6) Kelly, T. R.; De Silva, H.; Silva, R. A. Nature (London) 1999, 401,150 .

(7) Koumura, N.; Zijlstra, R. W. J.; van Delden, R. A.; Narada, N.; Feringa, B. L. Nature (London) 1999, 401, 152.

(8) Maxwell, J. C. Theory of Heat; Longmans, Green and Co.: London, 1871; Chapter 12. Also see: Leff, H. S.; Rex, A. F. Maxwell's Demon: Entropy, Information, Computing; Adam Hilger: Bristol, 1990.

(9) Loschmidt, J. Akad. Wiss. Wien Math.-Naturwiss. Kl. Sitzungsber. 1869, 59, Abth. 2, 395.

(10) Loschmidt, J. Akad. Wiss. Wien Math.-Naturwiss. Kl. Sitzungsber 1876, 73, Abth. 2, 128-142, 366.

(11) Flach, S.; Yevtushenko, O.; Zolotaryuk, Y. Phys. Rev. Lett. 2000, 84, 2358.

(12) Goychuk, I.; Hänggi, P. In Stochastic Processes in Physics, Chemistry, and Biology; Freund, J. A., Pöschel Th., Eds.; Lecture Notes in Physics 557; Springer: Berlin, 2000; pp 7-20.

(13) Dittrich, T.; Ketzmerick, R.; Otto, M.-F.; Schanz, H. Ann. Phys. (Leipzig) 2000, 9, 755.

(14) Ott, E. Chaos in Dynamical Systems; Cambridge University Press: Cambridge, 1993.

(15) Zaslavsky, G. M. Chaos 1995, 5, 653; Phys. Today 1999, 52 (8), 39.

(16) Goychuk, I.; Hänggi, P. Europhys. Lett. 1998, 43, 503.

(17) Dunlap, D. H.; Kenkre, V. M. Phys. Rev. B 1988, 37, 6622.

(18) Holthaus, M.; Hone, D. W. Philos. Mag. B 1996, 74, 105.

(19) Pronin, K. A.; Bandrauk, A. D.; Ovchinnikov, A. A. Phys. Rev. B 1994, $50,3473$.

(20) Goychuk, I.; Grifoni, M.; Hänggi, P. Phys. Rev. Lett. 1998, 81, 649; Phys. Rev. Lett. 1998, 81, 2837 (E).

(21) Van Kampen, N. G. Stochastic Processes in Physics and Chemistry; North-Holland: Amsterdam, 1992.

(22) Kubo, R. In Fluctuation, Relaxation and Resonance in Magnetic Systems; ter Haar, D., Ed.; Oliver and Boyd: Edinburgh, 1962; pp 23-68.

(23) Shapiro, V. E.; Loginov, V. M. Physica 1978, 91A, 563. Hänggi, P. Z. Physik 1978, B 31, 407 .

(24) Alekseev, K. N.; Erementchouk, M. V.; Kusmartsev, F. V. Europhys. Lett. 1999, 47, 595.

(25) Hänggi, P.; Talkner, P.; Borkovec, M. Rev. Mod. Phys. 1990, 62, 251.

(26) Weiss, U. Quantum Dissipative Systems. 2nd ed.; World Scientific: Singapore, 1999.

(27) Goychuk, I. A.; Petrov, E. G.; May, V. Phys. Rev. E 1995, 52, 2392.

(28) Hartmann, L.; Goychuk, I.; Hänggi, P. J. Chem. Phys. 2000, 113, 11159

(29) Gammaitoni, L.; Hänggi, P.; Jung, P.; Marchesoni, F. Rev. Mod. Phys. 1998, 70, 223.

(30) Berne, B. J.; De Leon, N.; Rosenberg, R. O. J. Phys. Chem. 1982, $86,2166$.

(31) De Leon, N.; Berne, B. J. Chem. Phys. Lett. 1982 93, 162; Chem. Phys. Lett. 1982, 93. 169. 